\title{
Electrical conduction and noise spectroscopy of sodium-alginate gold-covered ultrathin films for flexible green electronics
}

\section{Carlo Barone}

Dipartimento di Fisica "E.R. Caianiello", Università degli Studi di Salerno, Via Giovanni Paolo II 132, 84084 Fisciano (SA

\section{Piera Maccagnani}

CNR-Istituto per la Microelettronica e Microsistemi, Via P. Gobetti 101, 40129 Bologna

\section{Franco Dinelli}

CNR-Istituto Nazionale di Ottica, Via G. Moruzzi 1, 56124 Pisa

\section{Monica Bertoldo}

Dipartimento di Scienze Chimiche, Farmaceutiche ed Agrarie, Università degli Studi di Ferrara, Via L. Borsari 46, 44121 Ferrara

\section{Raffaella Capelli}

Dipartimento di Ingegneria E. Ferrari, Università di Modena e Reggio Emilia, 41125 Modena

\section{Massimo Cocchi}

CNR-Istituto per la Sintesi Organica e la Fotoreattività, Via P. Gobetti 101, 40129 Bologna

\section{Mirko Seri}

CNR-Istituto per lo Studio dei Materiali Nanostrutturati (ISMN), Via Piero Gobetti 101, 40129 Bologna Sergio Pagano ( $\sim$ spagano@unisa.it )

Dipartimento di Fisica "E.R. Caianiello", Università degli Studi di Salerno, Via Giovanni Paolo II 132, 84084 Fisciano (SA

\section{Research Article}

\section{Keywords:}

Posted Date: January 31st, 2022

DOI: https://doi.org/10.21203/rs.3.rs-1280895/v1

License: (c) (i) This work is licensed under a Creative Commons Attribution 4.0 International License. Read Full License 


\section{Abstract}

Green electronics is an emerging topic that requires the exploration of new methodologies for the integration of green components into electronic devices. Therefore, the development of alternative and eco-friendly raw materials, biocompatible and biodegradable, is of great importance. Among these, sodium-alginate is a natural biopolymer extracted from marine algae having a great potential in terms of transparency, flexibility, and conductivity, when functionalized with a thin gold ( $\mathrm{Au}$ ) layer. The electrical transport of these flexible and conducting substrates has been studied, by DC measurements, from 300 to $10 \mathrm{~K}$, to understand the interplay between the organic substrate and the metallic layer. The results were compared to reference bilayers based on polymethyl-methacrylate, a well-known polymer used in electronics. In addition, a detailed investigation of the electric noise properties was also performed. This analysis allows to study the effect of charge carriers fluctuations, providing important information to quantify the minimum metallic thickness required for electronic applications. In particular, the typical noise behavior of metallic compounds was observed in samples covered with $5 \mathrm{~nm}$ of $\mathrm{Au}$, while noise levels related to a non-metallic conduction were found for a thickness of $4.5 \mathrm{~nm}$, despite of the relatively good DC conductance of the bilayer.

\section{Introduction}

The development of flexible electronic devices has received much attention in the past decade, because they are expected to have a large impact in electrical and electronic equipment (EEE), which has become an essential part of our everyday life. After its use, EEE is disposed, generating large e-waste of hazardous but valuable materials. In 2019, the World generated 53,6 million metric tons (Mt) of e-waste, and only $17.4 \%$ of this e-waste was collected and recycled ${ }^{1}$. Present recycling technologies are mainly based on smelter and chemical technologies. The operation of a smelter is highly energy-intensive, while the commonly used mineral acids in chemical recycling techniques pose serious environmental risks for workers as well as for the air quality and water streams ${ }^{2,3}$.

The use of biodegradable and easily recyclable materials in the framework of green electronics could greatly reduce the environmental impact of e-waste. In fact, these materials offer the opportunity of a more environmentally sustainable recycling route ${ }^{2,4}$, as well as a safe management of single-use disposable devices such as sensors. According to European Standard EN13432, a biodegradable material is the one that can be converted by at least $90 \%$ into harmless components, such as water, carbon dioxide, and biomass, through the action of fungi or microorganisms within 6 months. Currently, the use of biodegradable materials represents an opportunity in several fields, where they can be used as substrates, inter- or active- layers and electrodes. To date, the most studied and promising biodegradable materials are cellulose derivatives ${ }^{5,6}$, chitin/chitosan ${ }^{7-9}$, and silk fibroin ${ }^{10-12}$.

Recently, we have started employing sodium-alginate (SA), a natural biodegradable polymer derived from brown algae ${ }^{13}$. SA is water soluble and easy to manipulate, so that flat and transparent foils can easily be fabricated with an environmentally friendly process ${ }^{14}$. With the aim of using SA for the fabrication of 
innovative substrates to produce green devices for light and energy (i.e. organic photodiodes (OPD), organic light emitting diodes (OLEDs), polymer solar cells (PSCs), etc.), we have deposited a thin Au layer on top of a SA film obtaining a conductive bilayer, whose electrical properties need to be studied in order to tailor the substrates to the final application. This platform has been successfully integrated in working OLEDs ${ }^{4}$, demonstrating that it can substitute the conventional one made of glass/ITO bilayers. Very thin metallic layers are however mandatory to preserve the substrate transparency in optoelectronic applications. Therefore, in the fabrication of a disposable EEE the amount of the metallic part in the bilayer should be minimal, while sustaining a proper current transport with a high electrical conductance. Au represents a good choice for its good growth and connectivity properties but also for its resistance to chemical degradation, providing a good conductance even at a very low thickness.

In this work, we have analyzed in detail the electrical transport in SA/Au substrates with Au thicknesses of few nanometers. It is very important to stress that, when investigating ultrathin films, a high conductance cannot be considered a sufficient property. Indeed, the Au film growth process on polymeric substrates, such as SA, proceeds in its first stages with the formation of nanoparticles only partially connected. The interconnections between the nanometric domains form for increasing Au thickness, until a full coverage of the surface is obtained ${ }^{15}$. This network morphology can lead to substrates with relatively high conductance but characterized by unstable conduction paths that may introduce large electric noise in real devices. The presence of fluctuating current paths can be readily evidenced performing a noise spectroscopy analysis, which allows determining the Au layer thickness that guarantees both a good electrical conductance and a low noise level.

\section{Results And Discussion}

\section{Electric transport and noise properties near the non-metallic threshold.}

As already mentioned, commercially available biopolymers, even if widely used and studied in various fields ranging from packaging to medicine, are poorly investigated in electronics and in particular as innovative green substrates. In this respect, a comparison of SA with a traditional material such as polymethyl-methacrylate (PMMA), whose properties and applications in electronic devices are wellknown, can provide interesting information.

In this type of noise characterizations, the main information is essentially given by the voltage-spectral density function $S_{V}$ and, more in details, by analyzing its amplitude frequency dependence. For the samples here investigated, the best fitting procedure of the spectral traces can be obtained by using a generic expression in the form of

$$
S_{V}(f)=\frac{K}{f^{\gamma}}+S_{0}
$$


Here, $\gamma$ is the noise frequency exponent, $S_{0}$ is a frequency-independent term, while $K$ is the noise amplitude coefficient whose study as a function of external parameters, such as temperature and bias current, allows establishing correlations and relationships with physical properties of the system involved ${ }^{18,19}$. The green curves in Fig. 2 show a good agreement between Eq. (1) and the experimental noise spectra, both for PMMA (left panel) and SA (right panel) Au coated films in the whole temperature range.

As result of the data analysis, varying the temperature from 300 to $10 \mathrm{~K}$, the exponent $\gamma$ ranges in the interval between 1.2 and 1.4 on both the investigated substrates. This suggests a small number $N$ of active fluctuators as responsible for the noise mechanisms ${ }^{18,20,21}$. In fact, a large number of Lorentzian fluctuators $(N \rightarrow \infty)$ would generate a pure $1 / \mathrm{f}$ noise component with $\gamma$ values ranging from 0.8 to $1.2^{18,22,23}$. The constant term $S_{0}$ is the "white-noise" component that essentially consists in the Johnson thermal noise $\left(4 k_{B} T R\right)$ added to a background contribution. Due to the small resistance values measured both for PMMA and for SA films, $S_{0}$ corresponds to the voltage-spectral density of the experimental setup electronic chain, being $\tilde{1} \times 10^{-18} \mathrm{~V}^{2} / \mathrm{Hz}$. The noise amplitude coefficient $K$, moreover, can be studied as a function of the applied bias current $I$, always revealing a quadratic behavior in the whole tested temperature range, as shown in Fig. 3. This is the expected standard behavior when the noise processes are originated by resistivity fluctuations in a random resistance network ${ }^{23,24}$.

Starting from the quadratic current dependence of $K$, it is straightforward to evaluate the Noise Level ( $N L$ ) of Ohmic systems as ${ }^{18}$

$$
N L=\frac{K}{V^{2}}=\frac{K}{R^{2} I^{2}}
$$

2

being $V$ the measured DC voltage. As evidenced in Fig. 4, a clear $N L$ peak occurs in the temperature region where an upturn of the resistance is observed. This happens around $128 \mathrm{~K}$ for PMMA (green circle) and around $112 \mathrm{~K}$ for SA (yellow circle). The presence of a peak in the noise level amplitude is usually associated to a change in the electric transport mechanisms ${ }^{25-28}$.

The transition to a typical non-metallic transport is confirmed by the increase of the normalized resistance $R / R_{300 \mathrm{~K}}$ below $100 \mathrm{~K}$ for Au film thickness $\leq 4.5 \mathrm{~nm}$. A comparison between the behavior on PMMA and SA shows minor differences indicating a minor role of the substrate composition on the $\mathrm{Au}$ growth. The ultrathin film can be regarded as a network of discontinuous metal regions instead of a continuous layer ${ }^{15}$, whose morphology does not depend on PMMA or SA and results in the specific nonmetallic features observed. From a technological point of view, the results of fluctuations spectroscopy give the indication of low $N L$ values in the low-temperature region, as expected because of the low charge carriers mobility. Moreover, similar low $N L$ values are observed even at high temperatures, in the range of a typical device use. This last feature is very interesting for the development of room- 
temperature applications. Notice that, while the general behavior of PMMA and SA spin-coated films is similar, the $N L$ value close to room temperature is lower on SA than PMMA, thanks to the peak shifted toward a lower temperature value in SA. This results in another advantage of SA over PMMA, in addition to the flexibility and the non-fossil-oil origin.

\section{Electric transport and noise properties above the metallic threshold.}

The evidence of a $N L$ peak, observed in the presence of a non-metallic conduction, completely disappears when the electric transport mechanisms change. In particular, increasing the thickness of the sputtered Au above $4.5 \mathrm{~nm}$, a more metallic behavior is recovered as shown in Fig. 5 for a free-standing SA film covered with a $5 \mathrm{~nm}$-thick Au layer. After a first thermal cycle (black squares, acquired in cooling mode) the polymeric matrix settles (red circles, acquired in warming mode), becoming more stable during the second thermal cycle (blue stars, acquired in cooling mode). After that, no hysteretic effect occurs. It is important to underline that, using Au pads (60 nm-thick, deposited onto the sample surface) for the electrical connections, the measurements are usually characterized by strong stability and repeatability. Signs of instability and non-repeatability are, instead, visible in absence of Au pads, as shown in Supplementary Fig. 1 where experimental data taken on Au spattered SA films with (red diamonds) and without (black circle) Au pads are compared.

Therefore, it is clear that a well-defined geometry of the contact pads allows an accurate evaluation of the intrinsic noise, as evidenced in Fig. 6for a typical metallized SA free-standing film (Au thickness of $5 \mathrm{~nm}$ ). More in details, the $1 / \mathrm{f}$ noise amplitude shows a decrease by lowering the temperature (see the twodimensional plot of Fig. 6a). The reduction of $K$ is usually expected in the case of metals, together with a quadratic current dependence of the $1 / f$ noise component ${ }^{18,22}$, as shown in the three-dimensional plot of Fig. $6 \mathrm{~b}$, which can be attributed to random resistance fluctuations.

From the literature it is known that this type of fluctuation processes, for metal or bad-metal compounds, is characterized by a noise level reduction for decreasing temperature ${ }^{29,30}$. This behavior is shown in Fig. 7 for all the PMMA and SA samples covered with a $5 \mathrm{~nm}$-thick Au layer, both for free-standing and spincoated films, whose typical metallic conduction is verified in Fig. 7a in terms of the normalized resistance $R / R_{300 K}$. In particular, Fig. 7b clearly evidences a monotonic decrease of $N L$ moving from 300 to $10 \mathrm{~K}$. One possible explanation for the observed noise temperature dependence can be found in a theoretical model which ascribes the origin of resistance fluctuations to vacancy and interstitial diffusion ${ }^{22,31}$, as already reported for granular and polycrystalline systems ${ }^{32,33}$. This finding gives an indication that above a certain Au layer thickness, here identified in $4.5 \mathrm{~nm}$, the conducting regions forming the ultrathin films are more uniformly distributed and interconnected, despite the possible presence of structural defects at the points of their closest distance. 
From the point of view of applications, also in the case of metallic films as already discussed for nonmetallic samples, it is important to stress that the intrinsic noise level is very low and has a weak dependence on the type of substrate used for the device fabrication. As a matter of fact, the free-standing

foils seems to generally have a lower $N L$ value compared to the compounds on glass, resulting very promising in the development of a flexible "green electronics".

\section{Conclusions}

The realization of green electronics may only start from the use of biopolymers, obtained from renewable resources, as innovative substrates. To date, despite several examples and promising results, the database of biomaterials for green devices needs to be expanded introducing new substances and compounds, and studying how their properties affect the performances and the stability of actual devices. To this end, substrates made of sodium-alginate, a natural biopolymer extracted from marine algae, covered with an ultrathin gold layer have been investigated from the electrical conduction point of view. In particular, a comparative study with polymethyl-methacrylate has been carried out on films spincoated on glass. The gold layer thickness has been kept to a minimum value in order to reduce the amount of expensive and non-biodegradable material employed while preserving good electrical conduction properties, although a possible recovery of the metals has been recently demonstrated for SAbased devices. A range between 4.5 and $5 \mathrm{~nm}$ has been identified as the region where the non-metallic and metallic transition occurs for both polymers. In addition, measurements have been performed on sodium-alginate free-standing films, highlighting its high potential for applications in flexible green electronics.

Measurements of DC resistivity, performed cycling the temperature from 300 to $10 \mathrm{~K}$, show that samples reach stability in the electrical conduction after one thermal cycle. Moreover, important information has been obtained by measuring the noise level amplitude, which is very low and comparable with metallic materials currently used in common electronics for samples with a $5 \mathrm{~nm}$-thick gold layer. For a thickness equal $4.5 \mathrm{~nm}$, the noise level amplitude is two orders of magnitude larger, indicating a completely different conduction mechanism, dominated by a random network of interconnected gold islands. This is important to define the minimum thickness of metallic layer to employ for the preparation of flexible transparent electrodes suitable for optoelectronic devices. Considering the broadest class of electronic applications, it must also be underlined the fact that a very thin layer of metallic material used for the functionalization of these bio-compounds is a critical aspect for disassembling and, subsequently, recovery and recycle of the precious components, in agreement with the concept of circular economy.

\section{Methods}

\section{Samples preparation.}

The SA solution ( $2 \%$ conc.) for spin-coating was prepared by solubilizing the appropriated amount of SA (Farmalabor Srl, Canosa di Puglia (BT), Italy) in ultrapure water under stirring at room temperature for 
several hours. The solution was left to stand at least overnight to allow bubbles escaping.

SA foils have been obtained casting a $4 \%$ water solution of pharmaceutical grade product (Farmalabor Srl, Canosa di Puglia (BT), Italy), into polystyrene Petri dishes ${ }^{15}$. Evaporation of the excess water is performed in clean room, under controlled environmental conditions (humidity $\sim 40 \%$, and temperature $\left.\sim 23^{\circ} \mathrm{C}\right)$. On free-standing samples, Au is sputtered in the central part of a rectangular SA stripe $(2.5 \times 1.5$ $\mathrm{cm}^{2}$ ), through a metal mask, using a MRC 8622 RF system. In order to have a fine control of Au thickness, the deposition processes were performed at $20 \mathrm{~W}$. A second Au deposition ( $60 \mathrm{~nm}$ thick), is executed for the fabrication of four pads, which guarantee good and stable Ohmic contact during the long electrical measurements.

On glass substrates ( $6 \mathrm{~mm}$ by side), SA and PMMA films were deposited by spin-coating in air. In detail, the SA solution ( $4 \% \mathrm{w} / \mathrm{w}$ ) was spin-coated at $2000 \mathrm{rpm}$ for $60 \mathrm{~s}$ (thickness of $\sim 400 \mathrm{~nm}$ ) and subsequently annealed in air at $80^{\circ} \mathrm{C}$ for $5 \mathrm{~min}$, while PMMA was spin-coated at $5000 \mathrm{rpm}$ for $60 \mathrm{~s}$ (thickness of $\sim 350 \mathrm{~nm}$ ) and subsequently annealed in air at $110^{\circ} \mathrm{C}$ for 3 hours. The thickness of the spincoated films was measured by a profilometer (KLATencor, P-6). For these samples the thin Au film is sputtered blanket on top of SA, applying the same deposition conditions used for free standing SA samples. Due to the reduced dimensions of these samples, no Au pads have been fabricated.

\section{Structural and morphological characterizations.}

Atomic force microscopy (AFM) was performed using a hybrid system made of a commercial head (SMENA, NT-MDT), home-built electronics and a digital lock-in amplifier (Zurich HF2LI). The setup was operated in intermittent contact mode (ICM). The cantilevers employed are commercially available from MikroMasch (HQ:NSC35). The imaging size of $2 \times 2 \mu \mathrm{m}^{2}$ has been chosen in order to observe the fine texture and to provide a sufficiently wide view.

The morphological analysis show that both the SA and PMMA films are very flat (Fig. 8), with a RMS roughness of 0.3 and $0.2 \mathrm{~nm}$, respectively. The SA films compared to the PMMA ones present a different texture with smaller features. All the Au films are also very flat, though rougher than the pristine films. The Au films on PMMA compared to SA present smaller grainy texture. The RMS values increase with increasing the Au thickness, with a maximum RMS roughness of $0.5 \mathrm{~nm}$ on SA and $0.6 \mathrm{~nm}$ on PMMA for an Au thickness of $5 \mathrm{~nm}$.

\section{Electrical transport and noise measurements.}

The electric transport characterizations of the samples under test were performed in a temperature stabilized closed-cycle refrigerator, mod. Janis CCS-350S (Lake Shore Cryotronics, Westerville, OH, USA), covering a wide range of temperature from 300 to $10 \mathrm{~K}$. Low noise DC and AC electronic bias and readout were used. In particular, the applied bias current was supplied with a DC current source, mod. Keithley 220 
(Tektronix, Beaverton, OR, USA). While, the AC signal was amplified with a home-made electronics, optimized for low-noise measurements ${ }^{34-36}$, and was acquired by a dynamic signal analyzer type HP35670A (Keysight Technologies, Santa Rosa, CA, USA).

Here, it is important to underline the fact that the background curves contain the relevant information and, therefore, the peaks visible in the voltage-noise spectra are not considered in the analysis, as they are due to external spurious sources. These unwanted noise contributions can also be generated by the contacts. In order to reduce such extrinsic components, two different types of electrical connections were made. The first one used two Au covered copper strips embedded in Kapton, placed at a distance of about 5 $\mathrm{mm}$, mechanically pressed onto the sample surface to obtain a four contacts geometry (see Fig. 9a, for details). Alternatively, a second type of contact configuration was realized by depositing four Au pads, 60 $\mathrm{nm}$-thick and $1 \mathrm{~mm}$-distant from each other, connected to the wires with silver paste (see Fig. 9b, for details). In all the cases, the contact noise was found to be negligible compared to the overall electric noise measured.

\section{Declarations}

\section{Data Availability}

The data that support the findings of this study are available from the corresponding authors upon reasonable request

\section{Competing interests}

The authors declare no competing interests.

\section{Author contributions}

C.B. and S.P. performed the electrical and noise measurements. P.M., F.D. and M.B. contributed to materials preparation and structural/morphological analysis tools. R.C., M.C. and M.S. developed the theoretical model. All authors analyzed and interpreted the data, and equally contributed to the paper writing. The submitted version of the manuscript was agreed by all.

\section{Acknowledgements}

The authors would like to thank S. Abate of CNR-SPIN Salerno (Italy) for his technical support. University of Salerno has partially supported this work through grants 300391FRB19PAGAN and 300391FRB20BARON. INFN is also gratefully acknowledged through experiments SIMP, Qub-IT, and DARTWARS.

\section{References}


1. Forti, V., Baldé, C. P., Kuehr, R. \& Bel, G. The Global E-waste Monitor 2020: Quantities, Flows, and the Circular Economy Potential. United Nations University (UNU)/United Nations Institute for Training and Research (UNITAR) - co-hosted SCYCLE Programme, International Telecommunication Union (ITU) \& International Solid Waste Association (ISWA), Bonn/Geneva/Rotterdam (United Nations University (UNU)/United Nations Institute for Training and Research (UNITAR) - co-hosted SCYCLE Programme, International Telecommunication Union (ITU) \& International Solid Waste Association (ISWA), 2020).

2. llyas, S., Srivastava, R. R., Kim, H., Das, S. \& Singh, V. K. Circular bioeconomy and environmental benignness through microbial recycling of e-waste: A case study on copper and gold restoration. Waste Manag. 121, 175-185 (2021).

3. Ahirwar, R. \& Tripathi, A. K. E-waste management: A review of recycling process, environmental and occupational health hazards, and potential solutions. Environ. Nanotechnology, Monit. Manag. 15, 100409 (2021).

4. Cocchi, M. et al. Fully Recyclable OLEDs Built on a Flexible Biopolymer Substrate. ACS Sustain. Chem. Eng. 9, 12733-12737 (2021).

5. Jung, Y. H. et al. High-performance green flexible electronics based on biodegradable cellulose nanofibril paper. Nat. Commun. 6, 7170 (2015).

6. Li, W. et al. Biodegradable Materials and Green Processing for Green Electronics. Adv. Mater. 32, 2001591 (2020).

7. Irimia-Vladu, M. "Green” electronics: biodegradable and biocompatible materials and devices for sustainable future. Chem. Soc. Rev. 43, 588-610 (2014).

8. Du, B.-W. et al. Eco-Friendly and Biodegradable Biopolymer Chitosan/Y203 Composite Materials in Flexible Organic Thin-Film Transistors. Materials vol. 101026 (2017).

9. Maddaloni, M., Vassalini, I. \& Alessandri, I. Green Routes for the Development of Chitin/Chitosan Sustainable Hydrogels. Sustainable Chemistry vol. 1 325-344 (2020).

10. Li, R.-W. \& Liu, G. Flexible and Stretchable Electronics. Flexible and Stretchable Electronics (Pan Stanford Publishing Pte Ltd, 2019). doi:10.1201/9780429058905.

11. Lee, C., Kim, S. \& Cho, Y.-H. Silk and Paper: Progress and Prospects in Green Photonics and Electronics. Adv. Sustain. Syst. n/a, 2000216 (2020).

12. Wen, D.-L. et al. Recent progress in silk fibroin-based flexible electronics. Microsystems Nanoeng. 7 , 35 (2021).

13. Rehm, B. H. A. \& Moradali, M. F. Alginates and Their Biomedical Applications. Alginates and Their Biomedical Applications (Springer, 2018). doi:10.1007/978-981-10-6910-9_1.

14. Pawar, S. N. \& Edgar, K. J. Alginate derivatization: A review of chemistry, properties and applications. Biomaterials 33, 3279-3305 (2012).

15. Maccagnani, P. et al. Flexible Conductors from Brown Algae for Green Electronics. Adv. Sustain. Syst. 3, 1900001 (2019). 
16. Barone, C. et al. Electric Transport in Gold-Covered Sodium-Alginate Free-Standing Foils. Nanomaterials 11, 565 (2021).

17. Sheng, P. Fluctuation-induced tunneling conduction in disordered materials. Phys. Rev. B 21, 21802195 (1980).

18. Kogan, S. Electronic Noise and Fluctuations in Solids. Electronic Noise and Fluctuations in Solids (Cambridge University Press, 1996). doi:10.1017/cbo9780511551666.

19. Bendat, J. S. \& Piersol, A. G. Random Data: Analysis and Measurement Procedures: Fourth Edition. Random Data: Analysis and Measurement Procedures: Fourth Edition (Wiley Blackwell, 2012). doi:10.1002/9781118032428.

20. Barone, C. et al. Probing transport mechanisms of BaFe2As2 superconducting films and grain boundary junctions by noise spectroscopy. Sci. Rep. 4, 6163 (2014).

21. Przybytek, J. et al. Robust random telegraph conductivity noise in single crystals of the ferromagnetic insulating manganite La0.86Ca0.14MnO3. Phys. Rev. B 95, 125101 (2017).

22. Dutta, P. \& Horn, P. M. Low-frequency fluctuations in solids: 1/f noise. Rev. Mod. Phys. 53, 497-516 (1981).

23. Hooge, F. N. 1/f Noise Sources. IEEE Trans. Electron Devices 41, 1926-1935 (1994).

24. Weissman, M. B. 1/f noise and other slow, nonexponential kinetics in condensed matter. Rev. Mod. Phys. 60, 537-571 (1988).

25. Chandni, U., Ghosh, A., Vijaya, H. S. \& Mohan, S. Criticality of Tuning in Athermal Phase Transitions. Phys. Rev. Lett. 102, 25701 (2009).

26. Chandni, U. \& Ghosh, A. Simple kinetic sensor to structural transitions. Phys. Rev. B 81, 134105 (2010).

27. Barone, C. et al. Unravelling the low-temperature metastable state in perovskite solar cells by noise spectroscopy. Sci. Rep. 6, 34675 (2016).

28. Pagano, S. et al. Iron-Based Superconducting Nanowires: Electric Transport and Voltage-Noise Properties. Nanomaterials 10, 862 (2020).

29. Dutta, P., Dimon, P. \& Horn, P. M. Energy Scales for Noise Processes in Metals. Phys. Rev. Lett. 43, 646-649 (1979).

30. Hooge, F. N., Kleinpenning, T. G. M. \& Vandamme, L. K. J. Experimental studies on 1/f noise. Reports Prog. Phys. 44, 479-532 (1981).

31. Stephany, J. F. Origin of $1 / f$ noise in metallic conductors and semiconductors. Phys. Rev. B 46, 12175-12180 (1992).

32. Barone, C. et al. Kondo-like transport and magnetic field effect of charge carrier fluctuations in granular aluminum oxide thin films. Sci. Rep. 8, 13892 (2018).

33. Di Trolio, A. et al. Transport mechanisms in Co-doped ZnO (ZCO) and H-irradiated ZCO polycrystalline thin films. Phys. Chem. Chem. Phys. 23, 2368-2376 (2021). 
34. Barone, C. et al. Experimental technique for reducing contact and background noise in voltage spectral density measurements. Rev. Sci. Instrum. 78, 093905 (2007).

35. Routoure, J. M., Wu, S., Barone, C., Méchin, L. \& Guillet, B. A Low-Noise and Quasi-Ideal DC Current Source Dedicated to Four-Probe Low-Frequency Noise Measurements. IEEE Trans. Instrum. Meas. 69, 194-200 (2020).

36. Barone, C. \& Pagano, S. What Can Electric Noise Spectroscopy Tell Us on the Physics of Perovskites? Coatings 11, 96 (2021).

\section{Figures}

\section{Figure 1}

Resistance versus temperature plots of non-metallic films. The data refer to three different investigated samples covered with a $4.5 \mathrm{~nm}$-thick Au layer: a) SA free-standing film (blue diamonds), b) PMMA deposited on glass (black squares), c) SA deposited on glass (red circles).

\section{Figure 2}

Voltage-noise spectra. The frequency dependence of , at a fixed bias current value, is shown for PMMA a) and for SA b) films deposited on glass and covered with a $4.5 \mathrm{~nm}$-thick Au layer. The green solid lines are the best fitting curves obtained by using Eq. (1).

\section{Figure 3}

Current dependence of the $1 / \mathrm{f}$ noise component. The amplitude of the $1 / \mathrm{f}$ noise is shown as a function of the applied bias current for PMMA a), b) and for SA c), d) non-metallic films. A typical quadratic behavior is always observed both below (upper panels) and above (lower panels) the temperatures at which a resistance minimum occurs for the two different investigated systems.

\section{Figure 4}

Comparison between DC and AC properties of non-metallic samples. The temperature dependencies of the normalized resistance $\mathbf{a}$ ) and of the Noise Level $\mathbf{b}$ ), as evaluated from Eq. (2), are shown for PMMA 
(squares) and SA (circles) films. The peaks, corresponding to the different resistance minima observed, are evidenced with green and yellow arrows for PMMA and SA, respectively.

\section{Figure 5}

Resistance versus temperature plots of metallic films. The temperature dependence of the resistance is shown for SA free-standing films covered with a $5 \mathrm{~nm}$-thick Au layer. The results, obtained on samples electrically contacted with Au pads, are reported for subsequent thermal cycles.

\section{Figure 6}

Noise properties of metallic samples. The amplitude of the $1 / \mathrm{f}$ noise component of SA free-standing films covered with a $5 \mathrm{~nm}$-thick Au layer is shown: a) as a function of temperature in a two-dimensional plot for different bias current values; $\mathbf{b}$ ) as a function of temperature and of bias current in a threedimensional plot.

\section{Figure 7}

Comparison between DC and AC properties of metallic samples. The temperature dependencies of the normalized resistance a) and of the Noise Level b) are shown for PMMA on glass (squares), SA on glass (circles), and SA free-standing (triangles) films. The typical characteristics of metallic compounds are observed, especially regarding the very low noise measured in the whole investigated temperature range.

\section{Figure 8}

Atomic force microscopy analysis. Morphological data of the Au films sputtered on SA (upper panels) and PMMA (lower panels) films supported on glass.

\section{Figure 9}

Electrical contacts configuration. a) Photograph of the sample holder used for measurements realized with mechanically pressed contact pads. b) Photograph of a typical investigated sample with inline Au pads deposited onto the surface for the electrical connections. 


\section{Supplementary Files}

This is a list of supplementary files associated with this preprint. Click to download.

- Supplementarylnformation.pdf

- Additionalinformation.docx 